\title{
Analysis of the activation status of Akt, NF $\kappa$ B, and Stat3 in human diffuse gliomas
}

\author{
Huamin Wang ${ }^{1}$, Hua Wang ${ }^{1}$, Wei Zhang ${ }^{1}$, Helen J Huang ${ }^{2}$, Warren SL Liao ${ }^{2}$ and \\ Gregory N Fuller ${ }^{1}$ \\ ${ }^{1}$ Department of Pathology and ${ }^{2}$ Department of Biochemistry and Molecular Biology, Program in Genes and \\ Development, The University of Texas MD Anderson Cancer Center, Houston, TX, USA
}

\begin{abstract}
Loss of phosphatase and tensin homolog (PTEN) and amplification of the epidermal growth factor receptor (EGFR) gene contribute to the progression of gliomas. As downstream targets of the PTEN and EGFR signaling pathways, Akt, NF $\kappa \mathrm{B}$, and signal transducer and activator of transcription-3 (Stat3) have been shown to play important roles in the control of cell proliferation, apoptosis, and oncogenesis. We examined the activation status of Akt, NFkB, and Stat3 in 259 diffuse gliomas using tissue microarrays and immunohistochemistry, and evaluated their association with glioma grade. We observed significant positive correlations between the activation status of Akt and $\mathrm{NF}_{\boldsymbol{k}} \mathrm{B}$ and glioma grade. In contrast, only focal immunoreactivity for phospho-Stat3 was observed in $<9 \%$ of high-grade gliomas. In addition, we observed a significant correlation between the activation of Akt and NF $\kappa$ B. Functional correlation between Akt activation and the activation of NF $\mathrm{B}^{\mathrm{B}}$ was confirmed in U251MG GBM cells in which inhibition of Akt activation either by stable expression of PTEN or by the PI3-kinase inhibitors, wortmannin and LY294002, led to a concomitant decrease in NF $k$ B-binding activity. Thus, our results demonstrate that constitutive activation of Akt and $\mathrm{NF} \kappa \mathrm{B}$, but not Stat3, contributes significantly to the progression of diffuse gliomas, and activation of Akt may lead to NF $\kappa$ B activation in highgrade gliomas.
\end{abstract}

Laboratory Investigation (2004) 84, 941-951, advance online publication, 7 June 2004; doi:10.1038/labinvest.3700123

Keywords: Akt; glioma; NFM $\kappa$ B; Stat3

Diffuse gliomas constitute the most common type of intracranial malignant neoplasm and account for more than $60 \%$ of all primary brain tumors. ${ }^{1}$ The World Health Organization (WHO) classification of tumors of the nervous system separates diffuse gliomas into seven principal categories: diffuse astrocytoma (LGA, WHO Grade II), oligodendroglioma (O, WHO Grade II), oligoastrocytoma (MOA, WHO Grade II), anaplastic astrocytoma (AA, WHO Grade III), anaplastic oligodendroglioma (AO, WHO Grade III), anaplastic oligoastrocytoma (AMOA, WHO Grade III), and glioblastoma (GBM, WHO Grade IV). ${ }^{1}$ Significant differences exist in patient survival between the different histologic grades of diffuse glioma. The median survival for LGA is greater than 5 years, compared to 2-5 years for AA, and less than 1 year for GBM. ${ }^{2-4}$

Correspondence: $\mathrm{Dr}$ GN Fuller, MD, PhD, Department of Pathology, Unit 085, The University of Texas MD Anderson Cancer Center, 1515 Holcombe Blvd, Houston, TX 77030, USA. E-mail: gfuller@mdanderson.org

Received 8 December 2003; revised and accepted 1 April 2004; published online 7 June 2004
Many alterations in the genes that regulate normal homeostasis of cell proliferation, differentiation, and apoptosis contribute to the formation and progression of diffuse gliomas. ${ }^{1}$ Among these genetic lesions, amplification of the epidermal growth factor receptor (EGFR) gene and mutation or deletion of the phosphatase and tensin homolog (PTEN) gene occur in $40-50 \%$ of high-grade gliomas, particularly in primary GBM. $^{5-8}$ EGFR is a transmembrane receptor with intrinsic tyrosine kinase activity that is normally regulated by ligands binding to its extracellular domain. In GBM, amplification of EGFR and inframe deletion of exons 2-7 of the EGFR gene, which results in a constitutively active form of EGFR, activate several signaling pathways, including the MAP kinase, PI3 kinase/Akt, and Jak/Stat pathways. $^{9,10}$

PTEN is a phospholipid phosphatase that dephosphorylates phosphatidylinositol 3,4,5-triphosphate. ${ }^{11-13}$ As such, PTEN inhibits PI3-kinasedependent activation of Akt. Conversely, mutation or loss of PTEN leads to constitutively activated Akt. In turn, activated Akt phosphorylates and inactivates Bad, caspase 9, and Forkhead transcription 
factors, which contribute not only to the suppression of apoptosis but also to the promotion of cell survival. ${ }^{14-16}$ PTEN is expressed at a significantly lower level in GBMs than in gliomas of lower grades. Independent of age and tumor grade, patients with gliomas expressing low levels of PTEN have a significantly poorer prognosis than do those with higher levels. ${ }^{17}$ PTEN has also been shown to regulate tumor-induced angiogenesis in GBM cell xenografts. ${ }^{18}$ Akt activity is elevated in GBM cells expressing mutant forms of PTEN. ${ }^{13}$ In animal studies, activation of Akt has been shown to be essential for the formation of GBMs from genetically modified neural progenitors and normal human astrocytes, suggesting that activation of Akt may play an important role in glioma formation and progression. ${ }^{19,20}$

Among many of its biological effects, activated Akt has been shown to phosphorylate and activate $\mathrm{I} \kappa \mathrm{B}$ kinase (IKK), which, in turn, phosphorylates $\mathrm{I} \kappa \mathrm{B}$ and leads to its degradation and to the nuclear translocation of $\mathrm{NF} \kappa \mathrm{B} .{ }^{21-23} \mathrm{NF} \kappa \mathrm{B}$ is a heterodimeric transcription factor consisting predominantly of p65/RelA and p50 subunits. $\mathrm{NF} \kappa \mathrm{B}$ is normally sequestered in the cytoplasm through its interaction with proteins belonging to the $\mathrm{I} \kappa \mathrm{B}$ family, which inhibits the translocation of $\mathrm{NF} \kappa \mathrm{B}$ into the nucleus. Stimulation by cytokines or growth factors leads to rapid phosphorylation of $\mathrm{I} \kappa \mathrm{B}$ by IKK. Phosphorylated $\mathrm{I} \kappa \mathrm{B}$ is rapidly ubiquinated and degraded through the 26S proteasome pathway. ${ }^{24}$ The degradation of $\mathrm{I} \kappa \mathrm{B}$ liberates $\mathrm{NF} \kappa \mathrm{B}$ and allows it to translocate into the nucleus where it regulates the transcription of target genes. ${ }^{24}$ It has also been shown that the p65 $\mathrm{NF} \kappa \mathrm{B}$ subunit is phosphorylated by IKK and by the PI3-kinase/Akt pathway, which leads to the activation of $\mathrm{NF} \kappa \mathrm{B}^{25-28}$ In human glioma cell lines, $\mathrm{NF} \kappa \mathrm{B}$ is constitutively activated and confers resistance to TNF- $\alpha$-induced apoptosis. $^{29,30}$ Conversely, inhibition of $\mathrm{NF} \kappa \mathrm{B}$ activation prevents cell cycle progression and inhibits the growth of U251MG glioma cells treated with TNF- $\alpha .{ }^{29} \mathrm{NF} \kappa \mathrm{B}$ has also been reported to be constitutively activated in other human cancers, including breast carcinoma, pancreatic adenocarcinoma, acute lymphoblastic leukemia, and high-grade gliomas, especially GBM. ${ }^{30-34}$ It has been speculated that constitutive activation of $\mathrm{NF} \kappa \mathrm{B}$ in GBM may be associated with tumor resistance to TNF- $\alpha$ immunotherapy. ${ }^{34}$ These findings suggest that $\mathrm{NF} \kappa \mathrm{B}$ activation could play an important role in the tumorigenesis of diffuse gliomas and in promoting the growth of high-grade gliomas. Previous studies on the activation of $\mathrm{Akt}$ and $\mathrm{NF} \kappa \mathrm{B}$ in diffuse gliomas, however, have been limited primarily to cultured cell lines or to relatively small numbers of tumors, and no large-scale studies have examined the activation of $\mathrm{Akt}$ and $\mathrm{NF} \kappa \mathrm{B}$ in human glioma tissues. It is also not clear whether activation of Akt correlates with the activation of $\mathrm{NF} \kappa \mathrm{B}$ in diffuse gliomas.
The signal transducer and activator of transcription (Stat) proteins constitute a family of latent transcription factors activated by a number of cytokines and growth factors, including epidermal growth factor (EGF) and interleukin-6. Stat proteins, particularly Stat3 and Stat5, have been reported to be constitutively activated in several cancers and cancer-derived cell lines. ${ }^{35}$ Despite the established connection between EGFR and Stat activation, the role of the Jak/Stat pathway in glioma progression is still not clear.

In this study, we used high-throughput tissue microarrays and immunohistochemistry to examine the activation status of Akt, $N F \kappa B$, and Stat 3 in 259 human diffuse gliomas, and correlated the results with glioma grade. We also examined the functional correlation between Akt activation and the activation of $\mathrm{NF} \kappa \mathrm{B}$ in diffuse gliomas. Furthermore, we examined the effect of inhibition of Akt on $\mathrm{NF} \kappa \mathrm{B}$ binding activities in U251MG GBM cells either by stable expression of PTEN or by the PI3-kinase inhibitors wortmannin and LY294002. This study provides the first dissection of two important signal transduction pathways in diffuse gliomas in vivo and offers new insights potentially useful for the design of mechanism-based therapies for GBM.

\section{Materials and methods}

\section{Stable Expression of PTEN in U251MG and LN229 GBM Cell Lines}

PT67 retrovirus producer cells were grown in Dulbecco's modified Eagle's medium/F12 containing $10 \%$ fetal calf serum, $1000 \mathrm{U} / \mathrm{ml}$ penicillinstreptomycin and $2 \mathrm{mM}$ glutamine, and transfected with pLNCX retrovirus harboring full-length PTEN cDNA by calcium phosphate precipitation. The LN229 and U251MG GBM cells were infected with 48-h supernatants from the transfected PT67 cells. The stable clones were selected with $400 \mu \mathrm{g} / \mathrm{ml} \mathrm{G418}$ and screened for PTEN expression by immunoblotting.

\section{Selection of Diffuse Glioma Samples and Construction of Glioma Tissue Microarray}

Glioma samples were obtained from patients who had undergone surgery at our institution from 1986 through 2001. The formalin-fixed, paraffinembedded archival tissue blocks were retrieved, and the matching hematoxylin and eosin (H\&E)-stained slides were reviewed and screened for representative tumor regions by a neuropathologist. Glioma samples were then grouped according to the diagnostic criteria of the WHO 2000 classification system. The composition of tumors included in the tissue microarray is listed in Table 1. A tissue microarray was constructed from the selected gliomas with a tissue microarrayer (Beecher 
Table 1 Composition of tumor types in glioma tissue microarray

\begin{tabular}{lc}
\hline Diffuse gliomas & $\begin{array}{c}\text { Number of } \\
\text { cases (tissue } \\
\text { cores) }\end{array}$ \\
\hline $\begin{array}{l}\text { Glioblastoma (GBM, WHO Grade IV) } \\
\text { Anaplastic astrocytoma (AA, WHO Grade III) }\end{array}$ & $70(140)$ \\
Diffuse astrocytoma (LGA, WHO Grade II) & $99(18)$ \\
Anaplastic oligodendroglioma (AO, WHO Grade III) & $41(82)$ \\
Oligodendroglioma (O, WHO Grade II) & $41(82)$ \\
Anaplastic mixed oligoastrocytoma (AMOA, WHO & $15(30)$ \\
Grade III) & \\
Oligoastrocytoma (MOA, WHO Grade II) & $24(48)$ \\
Gliosarcoma (GS, WHO Grade IV) & $10(20)$ \\
Normal brain (NB) & $10(20)$ \\
Control cell lines (CL) & $16(16)$
\end{tabular}

Instruments, Sun Prairie, WI, USA) as described previously. ${ }^{36}$ The tissue microarray included 259 primary gliomas representing all histologic types and grades of diffuse glioma codified in the WHO classification system. Each tumor was sampled in duplicate from representative areas of either one or two donor blocks using a $0.6-\mathrm{mm}$ punch, yielding a composite (array) block comprising a total of 555 tissue cores. In addition, 10 normal brain samples from surgical resections and 16 cultured cell lines (10 GBM, two breast cancer, two squamous cell carcinoma, one colon cancer, and one fetal kidney) were also included as negative and positive controls. Normal brain tissue samples were obtained from surgical resections. Each normal brain was sampled with two 0.6-mm tissue cores to include one core from normal white matter and one from gray matter. We specifically included LN229 and U251HF GBM cells that stably express PTEN as negative controls for pAkt immunohistochemical staining. U87MG and U373 GBM cells treated with TNF- $\alpha$ were included as a positive control for NF $\kappa$ B activation.

\section{Immunohistochemistry}

Anti-phospho-Akt ( $\mathrm{Ser}^{473}$ ), a polyclonal antibody that detects Akt only when phosphorylated at $\mathrm{Ser}^{473}$, anti-phospho-NF $\kappa \mathrm{B}$ p65 (Ser ${ }^{536}$ ) antibody, which is a polyclonal antibody that recognizes p65 only when phosphorylated at Ser ${ }^{536}$, and anti-phospho-Stat3, which is a monoclonal antibody that detects Stat3 only when it is phosphorylated at $\mathrm{Tyr}^{705}$, were purchased from Cell Signaling Technology (Beverly, MA, USA). Anti-NF $\kappa$ B p65 antibody, a polyclonal antibody directed against the $\mathrm{N}$-terminal domain of the human $\mathrm{NF} \kappa \mathrm{B}$ p65 subunit, was purchased from Santa Cruz Biotechnology (SC-109; Santa Cruz, CA, USA).

A standard indirect immunoperoxidase procedure (Elite-ABC kit; Vector Laboratories, Burlingame, CA, USA) was used for all staining. In brief, antigen retrieval was performed by treating the unstained slides in a steamer for $25 \mathrm{~min}$. Antibodies against phospho-Akt (pAkt, 1:50), $\mathrm{NF} \kappa \mathrm{B}$ p65 subunit (1:250), phospho-NF $\kappa$ B p65 $\left(\mathrm{Ser}^{536}\right)$ (pNF $\kappa \mathrm{B}$ p65, 1:50), or phospho-Stat3 (pStat3, 1:100) were overlaid on glioma array tissue sections and incubated overnight at $4^{\circ} \mathrm{C}$. Secondary antibody incubation was performed at room temperature for $60 \mathrm{~min}$. Mayer's hematoxylin nuclear staining was used as a counterstain. The results of immunohistochemical staining for pAkt, pStat $3, \mathrm{NF}_{\kappa} \mathrm{B}$, and $\mathrm{pNF} \kappa \mathrm{B}$ p65 subunit were evaluated independently by two pathologists and placed into one of two categories: positive or negative. The positive category represented gliomas exhibiting a strong positive reaction in at least $5 \%$ of the tumor cells in one tissue core, whereas the negative category represented tissue samples with very weak or absent staining. Cases with weak staining were always in less than $5 \%$ of the tumor cells.

\section{Western Blot}

Western blot analysis was used to examine the activation status of Akt and $\mathrm{NF} \kappa \mathrm{B}$ in GBM cells and frozen tissue samples from normal brains and GBMs. Briefly, U251MG cells were treated with control medium or with wortmannin or LY294002 for $3 \mathrm{~h}$ before harvest for cell extract preparation. All tissue samples used for Western blots were selected by neuropathologists from surgical resections based on frozen sections prepared from frozen tissue blocks. Equal amounts of proteins were resolved by $10 \%$ SDS-PAGE and electroblotted onto Hybond ECL nitrocellulose membranes (Amersham Pharmacia Biotech, Piscataway, NJ, USA). The filters were blocked in 5\% skim milk in $1 \times$ TBS and then probed with anti-Akt, anti-pAkt $\left(\mathrm{Ser}^{473}\right)$, or antipNF $\kappa$ B p65 (Ser $\left.{ }^{536}\right)$ antibodies and detected with an enhanced chemiluminescence kit (ECL, Amersham).

\section{NF $\kappa$ B DNA-Binding Assay}

Electrophoretic mobility shift assays (EMSA) were performed as described previously. ${ }^{37}$ Briefly, nuclear extracts were prepared from U251MG cells treated with control medium or with the PI3 kinase inhibitors wortmannin and LY294002. Nuclear proteins were incubated with radiolabeled $\mathrm{NF} \kappa \mathrm{B}$ binding oligonucleotides $\left(2 \times 10^{4}\right.$ c.p.m. $)$ as probe and nonspecific competitor poly(dI:dG). After incubation for $30 \mathrm{~min}$, protein-DNA complexes were resolved on $5 \%$ polyacrylamide gels and visualized by autoradiography.

\section{Statistical Analysis}

The results of immunohistochemical staining were analyzed using the $\chi^{2}$-test or Fisher's exact test, 
performed using the SigmaStat (version 2.0, SPSS, Chicago, IL, USA). $P<0.05$ is considered significant.

\section{Results}

Immunohistochemical staining for pAkt, $\mathrm{NF} \kappa \mathrm{B}$, $\mathrm{pNF} \kappa \mathrm{B}$ p65, and pStat3 in glioma tissue microarrays was technically successful in $98,95,98$, and $98 \%$ of the tumors, respectively. Representative micrographs of immunostaining for pAkt, $\mathrm{NF} \kappa \mathrm{B}$ p65, $\mathrm{pNF} \kappa \mathrm{B}$ p65, and pStat 3 in normal brain cortex and gliomas are shown in Figure 1. In normal brain tissues, pAkt was positive only in neurons, while $\mathrm{NF} \kappa \mathrm{B}$ and $\mathrm{pNF} \kappa \mathrm{B}$ p65 were positive only in endothelial cells. Astrocytes and oligodendrocytes were negative for $\mathrm{pAkt}, \mathrm{NF} \kappa \mathrm{B}$, and $\mathrm{pNF} \kappa \mathrm{B}$ p65 in all 10 normal brain samples examined (Figure 1a, c, and e). No immunoreactivity was observed for pStat3 in all 10 normal brains (Figure 1g). In gliomas, however, tumor cells showed diffuse cytoplasmic and scattered nuclear staining for pAkt, $\mathrm{NF} \kappa \mathrm{B}$ p65 and $\mathrm{pNF} \kappa \mathrm{B}$ p65. Immunostaining using anti-pStat3 antibody showed only focal nuclear staining in gliomas (Figure 1b, d, f, and h).

\section{Activation of Akt in Gliomas}

To determine whether correlation exists between activation of Akt and the histologic grade of glioma, the percentages of gliomas positive for pAkt in different histologic types and grades were determined and compared. As shown in Figure 2a, pAkt was positive in $84 \%(59 / 70), 44 \%(20 / 46)$, and $22 \%$ (2/9) of GBMs, AAs, and LGAs, respectively. Paired comparisons between GBMs and AAs, GBMs and LGAs, and AAs and LGAs showed statistically significant differences in the frequencies of Akt activation $(P<0.001)$. Similarly, comparisons between the anaplastic forms and the low-grade forms of the oligodendrocytic and the oligoastrocytic series also showed statistically significant differences in activation of Akt $(P<0.05)$ (Figure 2a). pAkt was overexpressed in 51\% (21/41) of AOs and $53 \%$ (8/15) of AMOAs, as compared to $18 \%(7 / 40)$ of Os and $8 \%(2 / 24)$ of MOAs. The frequencies of Akt activation in anaplastic groups of oligodendrocytic or oligoastrocytic tumors were significantly higher than those in Os or MOAs $(P<0.05)$. There were, however, no significant differences in Akt activation among AAs, AOs, and AMOAs, or among LGAs, Os, and MOAs. It is interesting that pAkt was also strongly positive in all gliosarcomas examined (10/ 10) (Figure 2a). Activated Akt was not observed in astrocytes or oligodendrocytes of normal brain cortex or cerebellum. To further confirm our immunohistochemical staining data, we performed Western blot analysis using frozen tissue samples from three normal brains and 14 GBMs. Of 14 GBMs, $10(71 \%)$ showed high levels of pAkt (Figure 3$)$. The other four GBMs also showed low, but detectable, levels of activated Akt. No pAkt was, however, detected in the normal brain tissues (Figure 3). These results show that activation of Akt significantly correlates with histologic grade in diffuse gliomas and the frequency of activated Akt increases with glioma progression.

\section{Overexpression and Activation of NF $\kappa B$ in Gliomas}

Immunostaining using anti-p65 antibody, which detects both the active and inactive $\mathrm{NF} \kappa \mathrm{B}$ p65 subunit, showed diffuse cytoplasmic staining with scattered nuclear staining in gliomas but was negative in the astrocytes and oligodendrocytes of normal brains. Using this antibody, $\mathrm{NF} \kappa \mathrm{B}$ p65 was shown to be overexpressed in $81 \%$ (56/69) of GBMs, $65 \%(32 / 49)$ of AAs, and 14\% (1/7) of LGAs. All 10 gliosarcomas also showed strong staining for $\mathrm{NF} \kappa \mathrm{B}$ p65 (Figure 2b). The difference in the frequency of $\mathrm{NF} \kappa \mathrm{B}$ p65 overexpression between GBMs and AAs was not statistically significant. High-grade astrocytomas showed a significantly higher frequency of $\mathrm{NF} \kappa \mathrm{B}$ overexpression when compared to LGAs $(P<0.05)$. NF $\kappa \mathrm{B}$ p65 was overexpressed in $77 \%$ (30/39) of AOs and 67\% (10/15) of AMOAs. Both of these expression rates were significantly higher than those of Os $(38 \%, 14 / 37)$ and MOAs $(38 \%, 8 / 21)$ (Figure 2b). Thus, these data show a strong positive correlation between overexpression of $\mathrm{NF} \kappa \mathrm{B}$ p65 and histologic grade in diffuse gliomas.

To determine whether elevated $\mathrm{NF} \kappa \mathrm{B}$ p65 in diffuse gliomas represents the active form of $\mathrm{NF} \kappa \mathrm{B}$, immunohistochemistry was performed using a polyclonal antibody directed against human $\mathrm{pNF} \kappa \mathrm{B}$ p65 (Figure 2b). This antibody detects activated $\mathrm{NF} \kappa \mathrm{B}$ p65 only when it is phosphorylated at $\operatorname{Ser}^{536} \cdot{ }^{25}$ pNF $\kappa$ B p65 was detected in $91.3 \%$ of GBMs, which was significantly higher than in anaplastic gliomas (AAs, 73.3\%; AO, 76.9\% ; and AMOA, 66.7\%), LGAs $(37.5 \%)$, Os $(50 \%)$, and MOAs $(50 \%)(P<0.05)$. The positive rates for $\mathrm{pNF} \kappa \mathrm{B}$ p65 in AAs and AOs were significantly higher than in LGAs, Os, and MOAs $(P<0.05)$. There was, however, no significant difference in $\mathrm{pNF} \kappa \mathrm{B}$ p65 among AAs, AOs and AMOAs, or among LGAs, Os and MOAs. Nine of 10 gliosarcomas examined were also positive for $\mathrm{pNF} \kappa \mathrm{B}$ p65 (Figure 2b). To further confirm the activation of $\mathrm{NF} \kappa \mathrm{B}$ in diffuse gliomas, Western blots were performed using the same antibody against $\mathrm{pNF} \kappa \mathrm{B}$ p65 on frozen tissue samples from normal brains and GBMs. The Western blots showed that pNF $\kappa \mathrm{B}$ p65 was detectable in $93 \%$ of the GBMs examined (13/14), but not in the three normal brain samples (Figure 3). Therefore, our data consistently show that $\mathrm{NF} \kappa \mathrm{B}$ is not only overexpressed but also activated in diffuse gliomas. Moreover, the degree of overexpression and activation of $\mathrm{NF} \kappa \mathrm{B}$ correlate with glioma grade, with high-grade gliomas showing higher levels of activated $\mathrm{NF} \kappa \mathrm{B}$ compared to lowgrade gliomas. 

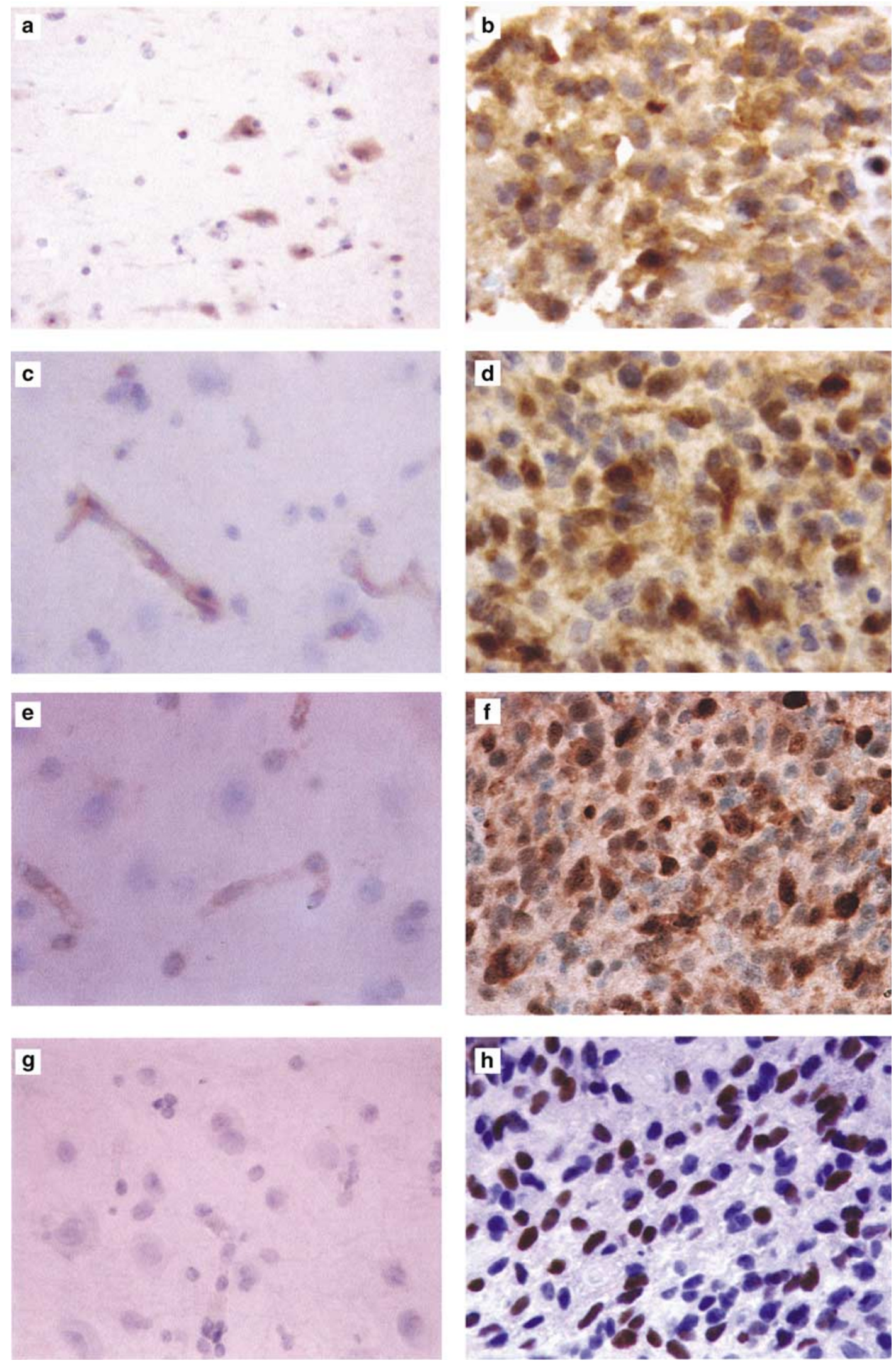

Figure 1 Expression of pAkt, $\mathrm{NF} \kappa \mathrm{B}$ p65, $\mathrm{pNF} \kappa \mathrm{B}$ p65, and pStat3 in normal brain cortex and gliomas. Representative micrographs of immunostaining are shown (original magnification, $\times 200$ ). Expression of pAkt in normal brain cortex (a) and GBM (b); expression of $\mathrm{NF} \kappa \mathrm{B}$ p65 in normal brain cortex (c) and GBM (d); expression of $\mathrm{pNF} \kappa \mathrm{B}$ p65 in normal brain cortex (e) and GBM (f); expression of pStat3 in normal brain cortex (g) and AO (h). 


\section{Expression of pStat3 in Gliomas}

Considering the well-established connection between the EGFR and Stat signaling pathways, we


Figure 2 Activation of Akt, $\mathrm{NF} \kappa \mathrm{B}$, and Stat3 in human gliomas. The percentage of positive tumors vs the histologic types and grades was plotted. (a) Activation of Akt correlates with histologic grade in diffuse gliomas. (b) Overexpression and activation of $\mathrm{NF} \kappa \mathrm{B}$ correlates with histologic grade in diffuse gliomas. (c) Focal expression of pStat 3 in diffuse gliomas. chose to examine the activation of Stat3 in gliomas using a monoclonal antibody that detects Stat3 only when phosphorylated at $\mathrm{Tyr}^{705}$. pStat 3 was detected in $9 \%(6 / 70), 8 \%(4 / 46)$, and $12.5 \%(1 / 8)$ of GBMs, AAs, and LGAs, respectively. Among the 254 gliomas for which data were available, only 23 cases (9\%) showed focally positive nuclear staining for pStat3. There was no correlation between histologic grade and pStat3 expression (Figure 2c). In contrast to the diffuse immunoreactivity seen for pAkt, $\mathrm{NF} \kappa \mathrm{B}$ p65, and $\mathrm{pNF}_{\kappa} \mathrm{B}$ p65, the immunoreactivity for pStat3 was focal in all 23 positive gliomas. In 17 of the 23 gliomas that showed positivity for pStat3, focally positive nuclear staining was present in only one of the two tissue cores from the same tumor.

\section{Activation of Akt in Diffuse Gliomas Contributes to the Activation of $\mathbf{N F} \boldsymbol{\kappa} B$}

When the activation status of Akt and $\mathrm{NF} \kappa \mathrm{B}$ was compared, a significant positive correlation was observed $(P<0.001$, Figure 4). Among the 122 gliomas that were positive for pAkt, $108(89 \%)$ also showed positive staining for activated $\mathrm{NF} \kappa \mathrm{B}$. In contrast, only $53 \%$ of the gliomas that were negative for $\mathrm{pAkt}$ showed positive staining for $\mathrm{pNF} \kappa \mathrm{B}$ (Figure 4). Consistent with this was the fact that 10 of 10 GBMs $(100 \%)$, which had high levels of pAkt, also showed increased levels of pNF $\kappa \mathrm{B}$ by Western blot (Figure 3). It is noteworthy that nine of 10 gliosarcomas that had elevated pAkt also showed positive staining for $\mathrm{pNF} \kappa \mathrm{B}$. A similar correlation between the expression of pAkt and $\mathrm{NF} \kappa \mathrm{B}$ was also obtained using antibody directed against $\mathrm{NF} \kappa \mathrm{B}$ p65 (data not shown). Therefore, our data demonstrate that activation of Akt may play a role in the activation of $\mathrm{NF} \kappa \mathrm{B}$ in high-grade gliomas.

To confirm whether activated Akt in high-grade gliomas contributes to the activation of $\mathrm{NF} \kappa \mathrm{B}$, we examined the activation status of Akt and $\mathrm{NF} \kappa \mathrm{B}$ in two GBM cell lines, U251MG and U251MG/PTEN, which were included in our glioma tissue microarray. U251MG/PTEN cells were derived from U251MG cells by the stable expression of PTEN. When probed with anti-pAkt, anti-NF $\kappa \mathrm{B}$ p65, or

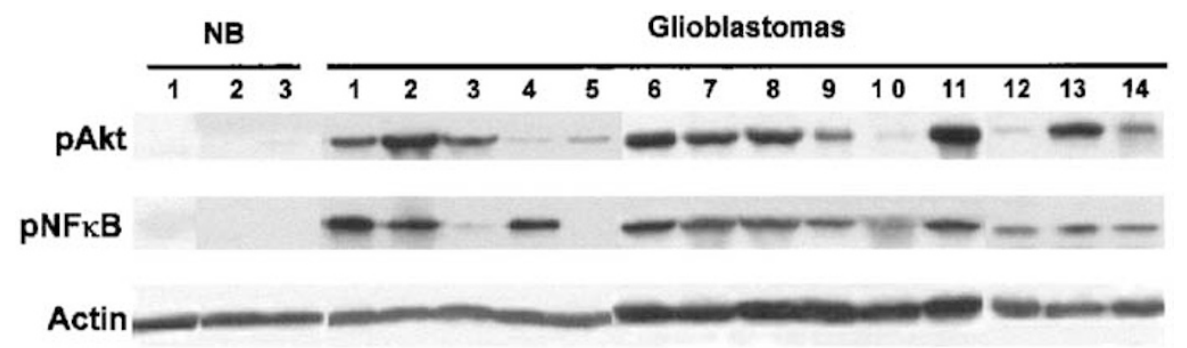

Figure 3 Activation of Akt and $\mathrm{NF} \kappa \mathrm{B}$ in normal brain and GBM. Tissue samples for normal brain and GBMs were selected by a neuropathologist based on examination of frozen sections prepared from frozen tissue blocks. Tissue extracts from normal brains (NB) and GBMs were Western blotted and probed with anti-pAkt (top panel), anti-pNF $\kappa$ B p65 (middle panel), and antibody against $\beta$-actin (bottom panel). 


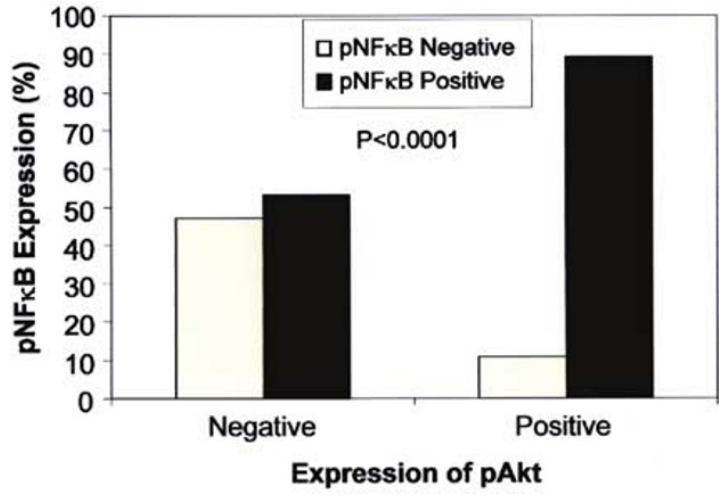

Figure 4 Expression of pAkt correlates with the expression of $\mathrm{pNF} \kappa \mathrm{B}$ p65 in diffuse gliomas.

anti-pNF $\kappa$ B p65 antibodies, U251MG cells showed an immunostaining pattern similar to that of glioma tissue samples: diffuse cytoplasmic staining with scattered nuclear staining (Figure 5a). The strong immunostaining for activated Akt and $\mathrm{pNF} \kappa \mathrm{B}$ p65 in U251 MG cells was confirmed by Western blotting of cell extracts using anti-pAkt antibody (Figure 5b) and by EMSA using ${ }^{32} \mathrm{P}$-labeled $\mathrm{NF} \kappa \mathrm{B}$ probes (Figure 5c). Expression of PTEN in U251MG/PTEN cells led to a marked decrease in activated Akt as determined by immunohistochemical staining (Figure 5a) or Western blotting (Figure 5b). Inhibition of Akt activation in U251MG/PTEN cells resulted in a concomitant decrease in NF $\kappa$ B DNA binding activity (Figure 5c). To further examine the role of activated Akt in the activation of $\mathrm{NF} \kappa \mathrm{B}$, two well-characterized PI3-kinase inhibitors, wortmannin and LY294002, were used to inhibit Akt, and the effect on $\mathrm{NF} \kappa \mathrm{B}$-binding activities was examined. Treatment of U251MG cells with wortmannin or LY294002 resulted in a dose-dependent decrease in the levels of pAkt (Figure 6a). These cells also showed a concomitant dose-dependent decrease in $\mathrm{NF} \kappa \mathrm{B}$-binding activities (Figure 6b). Similar results were obtained with U87MG and U373 cells (data not shown). These results strongly suggest that activated Akt in GBM cells contributes to the activation of $\mathrm{NF} \kappa \mathrm{B}$.

\section{Discussion}

Using tissue microarrays and immunohistochemistry, we examined 259 gliomas and found that both Akt and $\mathrm{NF}_{\kappa} \mathrm{B}$ were consistently activated in diffuse gliomas, but not in the astrocytes or oligodendrocytes of normal brain cortex and cerebellum. We observed a strong positive correlation between the activation status of Akt and $\mathrm{NF} \kappa \mathrm{B}$ and glioma histologic grade. These findings were confirmed by Western blot using frozen tissue from normal brains and GBMs. In contrast, focal nuclear immunoreactivity for pStat3 was observed only in a small percentage of gliomas examined. These findings indicate that activation of Akt and $\mathrm{NF}_{\kappa} \mathrm{B}$, but not Stat3, may play an important role in the formation and progression of human diffuse gliomas. In addition, we also observed a strong correlation between the activation of Akt and the activation of $\mathrm{NF} \kappa \mathrm{B}$, which was observed in high-grade glioma tissue samples and in GBM cell lines, suggesting that activation of Akt may lead to the activation of $\mathrm{NF} \kappa \mathrm{B}$ in gliomas.

The degree of heterogeneity and the expression pattern of the markers under study constitute a potential problem in tissue microarray-based studies, particularly in the case of GBM, a tumor whose molecular heterogeneity has been documented for many markers. The representativity of tissue microarray-based studies in general, however, has been extensively examined, and the data published so far have clearly indicated that the frequencies of molecular alterations identified by high-throughput tissue microarray screening analysis generally correspond well with those identified using full-size tissue sections. ${ }^{38}$ For example, good agreement among immunostaining results for p53 in diffuse gliomas has been shown in a comparison study of full-size tissue sections with tissue microarray analysis using single $0.6-\mathrm{mm}$ cores. ${ }^{39}$ To increase representativity, we have taken special precaution to select only representative tumor areas and incorporated two cores from each glioma sample into the tissue microarray. The concordant rates for immunostaining between the two tissue cores were $98 \%$ for pAkt and $94 \%$ for $\mathrm{NF} \kappa \mathrm{B}$ and $\mathrm{pNF} \kappa \mathrm{B}$ p65. Among 259 diffuse gliomas examined, only five cases (1.9\%; two GBMs, one AA, and two AOs) showed discordant expression of pAkt between the two cores. A slightly higher tissue core discordance rate was observed for $\mathrm{NF} \kappa \mathrm{B}$ immunostaining $(6 \%, 16 /$ 259). Discordant expression was observed only in high-grade gliomas. This may, in part, be due to the degree of heterogeneity in these tumors. For the cases with core discordance, the case was considered positive if one of the cores showed strong positive staining. In addition, we specifically included normal brain tissue cores and cytospin cores of genetically altered cells and cytokine-treated cells in our tissue microarray to serve as positive or negative controls. Such controls help to ensure the reliability of interpretation of the immunohistochemical data.

Akt, a serine/threonine kinase, belongs to a small gene family composed of three highly homologous members: Akt1, Akt2 and Akt3. The pAkt (Ser ${ }^{473}$ ) antibody used in this study detected all three forms, but only when they are phosphorylated at equivalent sites. Activation of Akt by the PI3-kinase pathway is inhibited by PTEN. U251MG/PTEN GBM cells, which stably express PTEN, showed negative immunostaining on our tissue microarray (Figure 5). U373 and U87MG, which contain mutations or deletions of PTEN, showed strong cytoplasmic and nuclear staining of pAkt (data not shown). 
U251MG

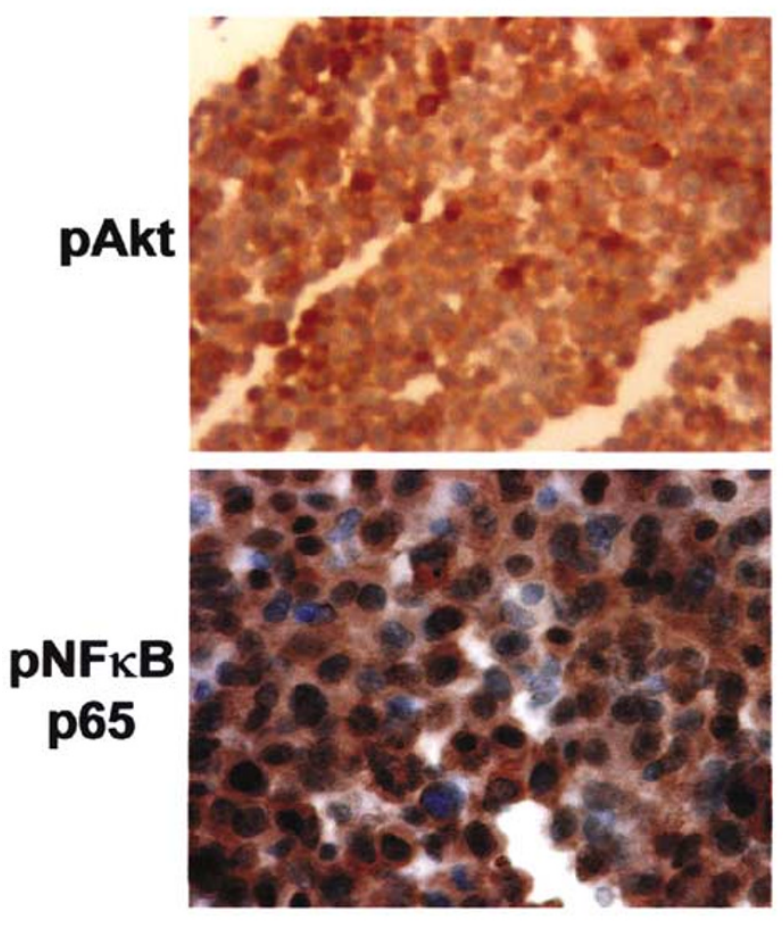

U251MG+PTEN
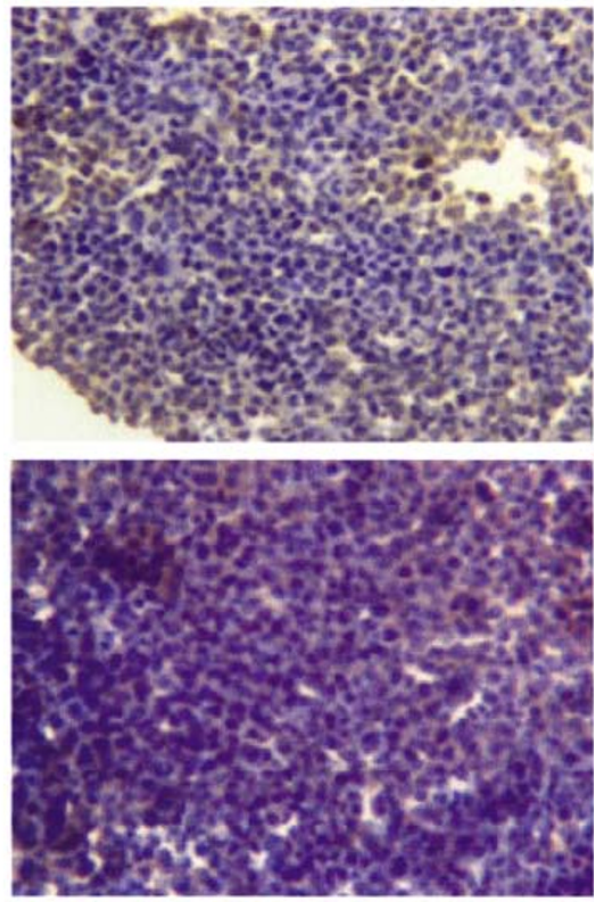

b

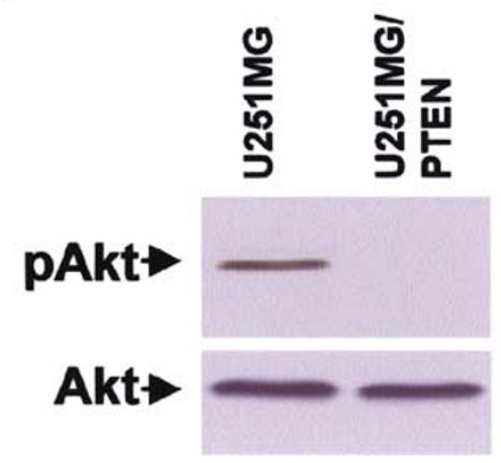

C

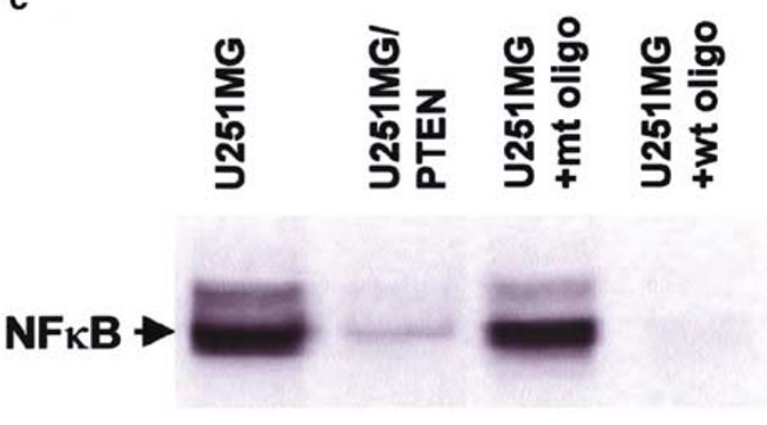

Figure 5 Expression of PTEN in U251MG cells inhibits Akt activation and NF $\kappa$ B binding activity. (a) Levels of pAkt and pNF $\kappa \mathrm{B}$ p65 are reduced in U251MG/PTEN cells. U251MG and U251MG/PTEN cells were stained with anti-pAkt and anti-pNF $\kappa$ B p65 antibodies. (b) U251MG/PTEN cells have reduced activated Akt. Cell extracts from U251MG and U251MG/PTEN cells were Western blotted and probed with anti-pAkt and anti-Akt antibodies. (c) U251MG/PTEN cells have reduced NF $\kappa$ B-binding activity. Nuclear extracts from U251MG and U251MG/PTEN cells were used in EMSA, with no competitor or with either wild-type (wt) or mutated (mt) NF $\kappa$ B-binding oligonucleotides (oligo). Position of the NF $\kappa \mathrm{B}$ :DNA complex is indicated by the arrow.

It is of interest that both cytoplasmic and nuclear staining was observed in glioma tissue samples. Nuclear translocation of Akt has been shown to be an important step in Akt-mediated cell proliferation and antiapoptosis. ${ }^{40-42}$ Our immunostaining results revealed statistically significant differences in the frequencies of pAkt expression among different grades of gliomas. In contrast, no pAkt immunostaining was detected in astrocytes or oligodendrocytes of 10 normal brain controls. Consistent with these findings is the fact that increased levels of pAkt were detected by Western blot in all 14 GBMs, but not in normal brains. Therefore, our data strongly suggest that activation of Akt correlates with glioma grade and support a role for Akt in glioma formation and progression.

Immunostaining for the $\mathrm{NF} \kappa \mathrm{B}$ p65 subunit with a polyclonal antibody that recognizes both active and inactive p65 showed diffuse cytoplasmic staining with scattered nuclear staining in gliomas. To further confirm the activation status of $\mathrm{NF} \kappa \mathrm{B}$ in diffuse gliomas, we performed immunohistochemical staining and Western blot analysis using a polyclonal anti-pNF $\kappa \mathrm{B}$ p65 antibody specific for activated $\mathrm{NF} \kappa \mathrm{B}$ p65. Phosphorylation of the p65 $\mathrm{NF} \kappa \mathrm{B}$ subunit by IKK occurs during the phospho- 



Figure 6 Wortmannin and LY294002 inhibit Akt activation and NF $\kappa$ B-binding activity in U251MG cells. (a) Cell extracts from control U251MG cells or cells treated for $3 \mathrm{~h}$ with wortmannin or LY294002 were probed with anti-pAkt or anti-Akt antibodies. (b) Nuclear extracts from cells treated as in (a) were incubated with ${ }^{32} \mathrm{P}$-labeled $\mathrm{NF} \kappa \mathrm{B}$-binding oligonucleotides in EMSA.

rylation and degradation of $\mathrm{I} \kappa \mathrm{Bs}$ and leads to the activation of $\mathrm{NF} \kappa \mathrm{B}^{25}$ Using this antibody, we observed a similar immunostaining pattern and a significantly higher frequency of $\mathrm{NF} \kappa \mathrm{B}$ activation in GBMs than in AAs and LGAs. No immunostaining was observed in astrocytes or oligodendrocytes in the 10 normal brain tissue samples included in our tissue microarray using either an anti-NF $\kappa \mathrm{B}$ p65 or an anti-pNF $\kappa \mathrm{B}$ p65 antibody. Therefore, our immunohistochemical staining data show that $\mathrm{NF} \kappa \mathrm{B}$ p65 is not only overexpressed but is also activated in diffuse gliomas compared to astrocytes or oligodendrocytes. This conclusion is further supported by increased expression of $\mathrm{pNF}_{\kappa} \mathrm{B}$ p65 in GBMs (93\%) by Western blot and increased $\mathrm{NF} \kappa \mathrm{B}$-binding activity in GBM cell lines. While it is unclear why most activated $\mathrm{NF} \kappa \mathrm{B}$ remains in the cytoplasm, Miyamoto et $a l^{43}$ have reported that only $10-20 \%$ of activated $\mathrm{NF} \kappa \mathrm{B}$ is located in the nucleus when $\mathrm{NF} \kappa \mathrm{B}$ is activated by long-term stimulation in differentiating $\mathrm{B}$ cells. The frequency of $\mathrm{NF} \kappa \mathrm{B}$ activation in anaplastic forms of gliomas was significantly lower than in GBMs. AAs and AOs (WHO grade III) had significantly higher frequencies of $\mathrm{NF} \kappa \mathrm{B}$ activation than grade II gliomas (LGA, Os, and MOAs). Therefore, our data show a strong correlation between the activation of $\mathrm{NF} \kappa \mathrm{B}$ and histologic grade of diffuse gliomas.

Stat3, a known downstream target of the EGFR signaling pathway, is activated in a variety of tumors. $^{35}$ Recently, activation of Stat3 in GBMs and LGAs has been reported using EMSA. ${ }^{44,45}$ Schaefer et $a l,{ }^{44}$ however, failed to demonstrate significant activation of Stat3 immunohistochemi- cally in glioma tumor cells. To address this inconsistency, we examined the activation of Stat3 in 259 human glioma samples of different grades using a monoclonal antibody that detects the active pStat3. We observed only focal nuclear staining for pStat3 in gliomas. Moreover, the positive rate was less than $9 \%$ in high-grade gliomas and was completely negative in all 10 gliosarcoma and 10 GBM cell lines examined. There was no correlation between Stat3 activation and histologic grade. This finding is consistent with the previous observation that failed to detect appreciable amounts of constitutively activated Stat $3 \alpha$ in eight untreated GBM cell lines. ${ }^{46}$ Therefore, our data do not support the notion that activation of Stat3 plays a significant role in glioma progression.

U251MG cells with high levels of activated Akt also showed high levels of $\mathrm{NF} \kappa \mathrm{B}$ binding activity (Figure 5). Phosphorylated Akt can activate $\mathrm{NF} \kappa \mathrm{B}$ either through activation of IKK or by direct phosphorylation of the $\mathrm{NF}_{\kappa} \mathrm{B}$ p65 subunit. ${ }^{21-23,26-28}$ The immunohistochemical data from the present tissue microarray study show a significant correlation between the activation of Akt and the activation of $\mathrm{NF} \kappa \mathrm{B}$ in diffuse gliomas. This correlation is further supported by Western blot analysis of frozen GBM tissue samples, which showed a $100 \%$ concordant expression rate for pAkt and $\mathrm{pNF}_{\kappa} \mathrm{B}$ p65. Our data are therefore consistent with the notion that activation of Akt may lead to activation of $\mathrm{NF} \kappa \mathrm{B}$ in diffuse gliomas. The functional importance of activated Akt in $\mathrm{NF} \kappa \mathrm{B}$ activation in human gliomas is further strengthened by the marked decrease in $\mathrm{NF} \kappa \mathrm{B}$-binding activity observed when 
U251MG cells are transfected with PTEN or treated with the PI3 kinase inhibitors, wortmannin and LY294002. As expected, U251MG/PTEN cells that express PTEN showed no detectable pAkt and were negative for pAkt by immunohistochemistry (Figure 5). The PTEN-expressing U251MG cell line also showed very low levels of $\mathrm{NF} \kappa \mathrm{B}$-binding activity. Furthermore, treatment of U251MG cells with the PI3 kinase inhibitors wortmannin and LY294002 not only reduced Akt activation, but also resulted in a concomitant decrease in $\mathrm{NF} \kappa \mathrm{B}$-binding activity. Therefore, our results show that activation of Akt plays a role in the activation of $\mathrm{NF} \kappa \mathrm{B}$ in gliomas. On the other hand, a significant number of gliomas that were negative for pAkt showed overexpression and activation of $\mathrm{NF} \kappa \mathrm{B}$, suggesting that other signaling pathways may also be involved in the activation of $\mathrm{NF} \kappa \mathrm{B}$ in diffuse gliomas.

The activation of both Akt and $\mathrm{NF} \kappa \mathrm{B}$ has been associated with antiapoptosis and cell proliferation in GBM cell lines and in animal studies. Activation of Akt and $\mathrm{NF} \kappa \mathrm{B}$ may lead to tumor resistance to TNF- $\alpha$, irradiation, or chemotherapy in GBM and other malignant gliomas. Thus, the ability to inhibit Akt and $\mathrm{NF} \kappa \mathrm{B}$ might confer an increased sensitivity to therapeutic modalities. The finding that activation of Akt and $\mathrm{NF} \kappa \mathrm{B}$ correlates with histologic grade in diffuse gliomas also offers potential avenues for the development of novel therapeutic strategies.

Finally, results of the present study demonstrate that molecular analyses performed on consecutive tissue microarray sections facilitate direct comparison of alterations of multiple molecular markers in nearly identical, histologically highly conserved tumor regions. The tissue microarray approach is thus well suited for rapid molecular dissection of oncogenetic regulatory pathways and subsequent correlation with histologic grade, tumor progression, and various important clinical characteristics. ${ }^{47}$

\section{References}

1 Cavenee WK, Furnari FB, Nagane M, et al. Diffusely infiltrating astrocytomas. In: Keilhues P, Cavenee WK (eds). Pathology \& Genetics: Tumors of the Nervous System. Lyon: IARC Press, 2000, pp 9-51.

2 Burger PC, Vogel FS, Green SB, et al. Glioblastoma multiforme and anaplastic astrocytoma. Pathologic criteria and prognostic implications. Cancer 1985; 56:1106-1111.

3 Daumas-Duport C, Scheithauer B, O'Fallon J, et al. Grading of astrocytomas. A simple and reproducible method. Cancer 1988;62:2152-2165.

$4 \mathrm{Kim}$ TS, Halliday AL, Hedley-Whyte ET, et al. Correlates of survival and the Daumas-Duport grading system for astrocytomas. J Neurosurg 1991;74:27-37.

5 Wang SI, Puc J, Li J, et al. Somatic mutations of PTEN in glioblastoma multiforme. Cancer Res 1997;57: 4183-4186.
6 Tohma Y, Gratas C, Biernat W, et al. PTEN (MMAC1) mutations are frequent in primary glioblastomas (de novo) but not in secondary glioblastomas. J Neuropathol Exp Neurol 1998;57:684-689.

7 Teng DH, Hu R, Lin H, et al. MMAC1/PTEN mutations in primary tumor specimens and tumor cell lines. Cancer Res 1997;57:5221-5225.

8 Steck PA, Lin H, Langford LA, et al. Functional and molecular analyses of $10 \mathrm{q}$ deletions in human gliomas. Genes Chromosomes Cancer 1999;24:135-143.

9 Batra SK, Castelino-Prabhu S, Wikstrand CJ, et al. Epidermal growth factor ligand-independent, unregulated, cell-transforming potential of a naturally occurring human mutant EGFRvIII gene. Cell Growth Differ 1995;6:1251-1259.

10 Schwechheimer K, Huang S, Cavenee WK. EGFR gene amplification-rearrangement in human glioblastomas. Int J Cancer 1995;62:145-148.

11 Myers MP, Pass I, Batty IH, et al. The lipid phosphatase activity of PTEN is critical for its tumor supressor function. Proc Natl Acad Sci USA 1998;95: 13513-13518.

12 Maehama T, Dixon JE. The tumor suppressor, PTEN/ MMAC1, dephosphorylates the lipid second messenger, phosphatidylinositol 3,4,5-trisphosphate. J Biol Chem 1998;273:13375-13378.

13 Haas-Kogan D, Shalev N, Wong M, et al. Protein kinase $\mathrm{B}$ (PKB/Akt) activity is elevated in glioblastoma cells due to mutation of the tumor suppressor PTEN/ MMAC. Curr Biol 1998;8:1195-1198.

14 Datta SR, Dudek H, Tao X, et al. Akt phosphorylation of BAD couples survival signals to the cell-intrinsic death machinery. Cell 1997;91:231-241.

15 Cardone MH, Roy N, Stennicke HR, et al. Regulation of cell death protease caspase-9 by phosphorylation. Science 1998;282:1318-1321.

16 Brunet A, Bonni A, Zigmond MJ, et al. Akt promotes cell survival by phosphorylating and inhibiting a Forkhead transcription factor. Cell 1999;96:857-868.

17 Sano T, Lin H, Chen X, et al. Differential expression of MMAC/PTEN in glioblastoma multiforme: relationship to localization and prognosis. Cancer Res 1999; 59:1820-1824.

18 Wen S, Stolarov J, Myers MP, et al. PTEN controls tumor-induced angiogenesis. Proc Natl Acad Sci USA 2001;98:4622-4627.

19 Holland EC, Celestino J, Dai C, et al. Combined activation of Ras and Akt in neural progenitors induces glioblastoma formation in mice. Nat Genet 2000;25: 55-57.

20 Sonoda Y, Ozawa T, Aldape KD, et al. Akt pathway activation converts anaplastic astrocytoma to glioblastoma multiforme in a human astrocyte model of glioma. Cancer Res 2001;61:6674-6678.

21 Ozes ON, Mayo LD, Gustin JA, et al. NF-kappaB activation by tumour necrosis factor requires the Akt serine-threonine kinase. Nature 1999;401:82-85.

22 Romashkova JA, Makarov SS. NF-kappaB is a target of AKT in anti-apoptotic PDGF signalling. Nature 1999;401:86-90.

23 Gustin JA, Maehama T, Dixon JE, et al. The PTEN tumor suppressor protein inhibits tumor necrosis factor-induced nuclear factor kappa B activity. J Biol Chem 2001;276:27740-27744.

24 Mercurio F, Manning AM. Multiple signals conver ging on NF-kappaB. Curr Opin Cell Biol 1999;11: 226-232. 
25 Sakurai H, Chiba H, Miyoshi H, et al. IkappaB kinases phosphorylate NF-kappaB p65 subunit on serine 536 in the transactivation domain. J Biol Chem 1999;274:30353-30356.

26 Mayo MW, Madrid LV, Westerheide SD, et al. PTEN blocks tumor necrosis factor-induced NF-kappa Bdependent transcription by inhibiting the transactivation potential of the p65 subunit. J Biol Chem 2002;277:11116-11125.

27 Sizemore N, Lerner N, Dombrowski N, et al. Distinct roles of the Ikappa B kinase alpha and beta subunits in liberating nuclear factor kappa B (NF-kappa B) from Ikappa B and in phosphorylating the p65 subunit of NF-kappa B. J Biol Chem 2002;277:3863-3869.

28 Koul D, Yao Y, Abbruzzese JL, et al. Tumor suppressor MMAC/PTEN inhibits cytokine-induced NFkappaB activation without interfering with the IkappaB degradation pathway. J Biol Chem 2001;276:11402-11408.

29 Otsuka G, Nagaya T, Saito K, et al. Inhibition of nuclear factor-kappaB activation confers sensitivity to tumor necrosis factor-alpha by impairment of cell cycle progression in human glioma cells. Cancer Res 1999; 59:4446-4452.

30 Nagai S, Washiyama K, Kurimoto M, et al. Aberrant nuclear factor-kappaB activity and its participation in the growth of human malignant astrocytoma. J Neurosurg 2002;96:909-917.

31 Sovak MA, Bellas RE, Kim DW, et al. Aberrant nuclear factor-kappaB/Rel expression and the pathogenesis of breast cancer. J Clin Invest 1997;100:2952-2960.

32 Wang W, Abbruzzese JL, Evans DB, et al. The nuclear factor-kappa B RelA transcription factor is constitutively activated in human pancreatic adenocarcinoma cells. Clin Cancer Res 1999;5:119-127.

33 Kordes U, Krappmann D, Heissmeyer V, et al. Transcription factor NF-kappaB is constitutively activated in acute lymphoblastic leukemia cells. Leukemia 2000;14:399-402.

34 Yamamoto M, Fukushima T, Hayashi S, et al. Correlation of the expression of nuclear factor-kappa B, tumor necrosis factor receptor type 1 (TNFR 1) and c-Myc with the clinical course in the treatment of malignant astrocytomas with recombinant mutant human tumor necrosis factor-alpha (TNF-SAM2). Anticancer Res 2000;20:611-618.

35 Buettner R, Mora LB, Jove R. Activated STAT signaling in human tumors provides novel molecular targets for therapeutic intervention. Clin Cancer Res 2002;8:945-954.
36 Kononen J, Bubendorf L, Kallioniemi A, et al. Tissue microarrays for high-throughput molecular profiling of tumor specimens. Nat Med 1998;4:844-847.

37 Reddy SA, Huang JH, Liao WS. Phosphatidylinositol 3-kinase in interleukin 1 signaling. Physical interaction with the interleukin 1 receptor and requirement in NFkappaB and AP-1 activation. J Biol Chem 1997;272: 29167-29173.

38 Wang H, Zhang W, Fuller GN. Tissue microarrays: applications in neuropathology research, diagnosis, and education. Brain Pathol 2002;12:95-107.

39 Sallinen SL, Sallinen PK, Haapasalo HK, et al. Identification of differentially expressed genes in human gliomas by DNA microarray and tissue chip techniques. Cancer Res 2000;60:6617-6622.

40 Meier R, Alessi DR, Cron P, et al. Mitogenic activation, phosphorylation, and nuclear translocation of protein kinase Bbeta. J Biol Chem 1997;272:30491-30497.

41 Pekarsky Y, Koval A, Hallas C, et al. Tcl1 enhances Akt kinase activity and mediates its nuclear translocation. Proc Natl Acad Sci USA 2000;97:3028-3033.

42 Borgatti P, Martelli AM, Bellacosa A, et al. Translocation of $\mathrm{Akt} / \mathrm{PKB}$ to the nucleus of osteoblast-like MC3T3-E1 cells exposed to proliferative growth factors. FEBS Lett 2000;477:27-32.

43 Miyamoto S, Chiao PJ, Verma IM. Enhanced I kappa B alpha degradation is responsible for constitutive NFkappa B activity in mature murine B-cell lines. Mol Cell Biol 1994;14:3276-3282.

44 Schaefer LK, Ren Z, Fuller GN, et al. Constitutive activation of Stat3alpha in brain tumors: localization to tumor endothelial cells and activation by the endothelial tyrosine kinase receptor (VEGFR-2). Oncogene 2002;21:2058-2065.

45 Rahaman SO, Harbor PC, Chernova O, et al. Inhibition of constitutively active Stat3 suppresses proliferation and induces apoptosis in glioblastoma multiforme cells. Oncogene 2002;21:8404-8413.

46 Schaefer LK, Menter DG, Schaefer TS. Activation of stat3 and stat1 DNA binding and transcriptional activity in human brain tumour cell lines by gp130 cytokines. Cell Signal 2000;12:143-151.

47 Wang $\mathrm{H}$, Wang $\mathrm{H}$, Zhang $\mathrm{W}$, et al. Overexpression of insulin-like growth factor binding protein 2 correlates with un-regulation of vascular endothelial growth factor in human diffuse gliomas: an immunohistochemical tissue microarray study of 259 tumors. Neuro-oncology 2001;3:271-272. 